\title{
APLICABILIDADE PRÁTICA DA CARTILHA “PUNÇÃO VENOSA PERIFÉRICA PARA FAMÍLIA”
}

\author{
Cleonara Sousa Gomes e Silva ${ }^{1}$; Silvone Santa Barbara da Silva Santos²; Luciano \\ Marques dos Santos ${ }^{3}$ e Sarah Almeida Santos ${ }^{4}$ \\ 1. Bolsista PIBIC/CNPq, Graduanda em Enfermagem, Universidade Estadual de Feira de Santana, e-mail: \\ cleosilvauefs@gmail.com
}

2. Orientadora, Departamento de Saúde Universidade Estadual de Feira de Santana, e-mail: silvone.santabarbara@gmail.com

3. Coordenador do projeto Segurança do paciente pediátrico e sua família: estudo de tecnologias e evento adverso associado a terapia intravascular periférica, Departamento de Saúde, Universidade Estadual de Feira de Santana, e-mail: lucmarxenfo@yahoo.com.br

4. Participante do projeto Segurança do paciente pediátrico e sua família: estudo de tecnologias e evento adverso associado a terapia intravascular periférica, Departamento de Saúde, Universidade Estadual de Feira de Santana, e-mail: sarahsantosuefs@gmail.com

\begin{abstract}
PALAVRAS-CHAVE: Enfermagem Pediátrica; Tecnologia Educacional; Cateterismo venoso periférico.
\end{abstract}

\section{INTRODUÇÃO}

As experiências vivenciadas pelos familiares acompanhantes durante a hospitalização da criança são diversas vezes causadoras de muito sofrimento e estresse. Segundo Bezerra et al (2009); Conceição et al (2011) a Cateterização Intravenosa Periférica (CIP) em crianças é um dos procedimentos invasivos e dolorosos que produz maior sofrimento para os familiares.

Os sentimentos apresentados por eles são ansiedade, nervosismo, medo e tristeza, manifestando-se reações como choro, atitudes agressivas e vontade de sair da enfermaria durante o procedimento. (Lisboa, 2014; Conceição et al. 2011; Bezerra et al. 2009)

A introdução de materiais didáticos e instrucionais e métodos educativos denotam uma importante ferramenta assistencial na promoção do cuidado ao familiar, segundo a filosofia do Cuidado Centrado na Família e na Criança (CCFC).

Um exemplo disso é a cartilha "Punção venosa periférica para família" elaborada para os acompanhantes de crianças hospitalizadas que foram submetidas a CIP com o finalidade de facilitar a compreensão do público sobre a temática, além de direcioná-los quanto as estratégias que podem ser adotadas durante o procedimento para tranquilizar a eles e as crianças.

Diante disso, o objetivo desse estudo é verificar a aplicabilidade prática da cartilha "Punção venosa periférica para família" junto aos familiares acompanhantes de crianças hospitalizadas em um hospital pediátrico de Feira de Santana.

Com relação ao interesse pelo estudo, esta relacionado ao estado da arte estabelecido, no período de outubro a dezembro de 2015, sendo percebida a pouca produção de estudos sobre essa temática em periódico de circulação nacional e internacional: Biblioteca Virtual em Saúde (BVS), PubMed, Science Direct, SCOPUS, Web of Science e CUIDEN. Os descritores em saúde utilizados forma: Família, Enfermagem Pediátrica, Enfermagem Familiar, Criança Hospitalizada, Tecnologia Educacional, Material de Ensino, Cateterismo Periférico, Segurança do Paciente, Infusões Intravenosas e Estudos de Validação, além de seus correspondentes em inglês e espanhol, e os semelhantes do Medical Subject Headings (MESH).

Isto posto, questiona-se a Cartilha "Punção venosa periférica para família” tem aplicabilidade prática? 


\section{MATERIAL E MÉTODOS}

Trata-se de um estudo metodológico do tipo elaboração de tecnologia, realizado em 2016. Para verificar a aplicabilidade prática da cartilha participaram do estudo 44 familiares acompanhantes de crianças hospitalizadas que já tenham visto a CIP nas crianças, sendo realizada em um hospital estadual pediátrico de Feira de Santana. Utilizou-se da técnica Delphi para nortear a coleta de dados (Scarparo et al., 2012). Foram avaliadas as seguintes categorias referentes ao material: conteúdo, linguagem, aparência e motivação. Adotou-se como consenso das respostas o Índice de Validação de Conteúdo (IVC) com valor igual ou superior a 0,70 (Perroca, 2011; Scarparo et al., 2012; Avelar; Pedreira; Peterlini, 2013). Este estudo foi aprovado pelo Comitê de Ética na Pesquisa da Universidade Estadual de Feira de Santana através do parecer 841612.

\section{RESULTADOS}

A cartilha "Punção venosa periférica para família" obteve aplicabilidade prática, de açodo com a avaliação feita pelos familiares do estudo. Sendo observado que o IVC foi maior que 0,70 em todas as variáveis. Nas tabelas 1 e 2 apontam os índices apresentados nas categorias sobre conteúdo, linguagem, aparência e motivação.

Tabela 1 - Distribuição do Índice de Concordância e Validade de Conteúdo com os Familiares segundo critério de conteúdo e linguagem da primeira rodada. Feira de Santana (BA), 2016.

\begin{tabular}{|c|c|}
\hline \multirow[t]{2}{*}{ Variáveis } & ${ }^{*} \mathrm{IVC}$ \\
\hline & Individual \\
\hline \multicolumn{2}{|l|}{ 1. Conteúdo } \\
\hline 1.1 Os sentimentos apresentados pelos familiares da cartilha estão de acordo; & 1,0 \\
\hline 1.20 conceito de punção venosa está claro & 0,97 \\
\hline 1.30 conceito de veia está claro & 0,97 \\
\hline 1.40 conceito de cateter está claro & 0,97 \\
\hline 1.50 passo a passo do procedimento está claro & 0,97 \\
\hline 1.6 As dicas do que fazer durante o procedimento estão claras & 0,95 \\
\hline$* *$ IAP Global & 0,97 \\
\hline \multicolumn{2}{|l|}{ 2. Linguagem } \\
\hline 2.1 As frases são de fácil entendimento & 1,0 \\
\hline 2.2 A escrita utilizada está atrativa & 1,0 \\
\hline$\therefore$ *IAP Global & 1,0 \\
\hline
\end{tabular}

Fonte: Coleta de Dados

*IVC: Índice de Validação de Conteúdo

**IVC Global: Índice de Validação de Conteúdo Global

A cartilha possui boa aplicabilidade prática, segundo avaliação dos familiares. No entanto, foram propostas algumas sugestões, visando melhor o entendimento do material, como informações mais objetivas.

\section{DISCUSSÃO}

A utilização de tais tecnologias sobre CIP para familiares de crianças hospitalizadas pode contribuir com a compreensão deles sobre a temática, por conseguinte, pode influenciar nas ações e sentimentos manifestados pelos familiares durante o procedimento, através do desenvolvimento de estratégias que minimizem o estresse e o sofrimento (Won, 2006; Benchaya; Ferreira; Brasiliense, 2014).

Desse modo, as atitudes expressadas pelos pais que fizeram uso de materiais e métodos educativos também repercuti nas reações apresentadas pelas crianças (Grupta et al. 2014; Mahoney, Ayers e Seddon, 2010; Spagrud et al. 2008; Won, 2006). 
No estudo feito por Won (2006) observou-se que os familiares que faziam uso do programa de informação tinham maiores índices de apoio a criança, além de eles manifestarem diminuição significativa dos sentimentos negativos.

Tabela 2 - Distribuição do Índice de Concordância e Validação de Conteúdo com os Familiares segundo critério de aparência e motivação. Feira de Santana (BA), 2016.

\begin{tabular}{|c|c|}
\hline Variáveis & ${ }^{*}$ IVC \\
\hline & Individual \\
\hline \multicolumn{2}{|l|}{ 3. Aparência } \\
\hline 3.1 A capa chama atenção; & 1,0 \\
\hline 3.2 A capa mostra o assunto a que se refere: & 0,72 \\
\hline 3.3 A sequência do tema está adequada; & 0,97 \\
\hline 3.4 O tamanho do conteúdo em cada página está adequado: & 0,90 \\
\hline 3.5 O tamanho das letras é adequado; & 1,0 \\
\hline 3.6 As ilustrações são claras e facilitam a aprendizagem; & 0,97 \\
\hline 3.7 O número de ilustrações facilita o entendimento; & 0,95 \\
\hline$* *$ IAP Global & 0,93 \\
\hline \multicolumn{2}{|l|}{ 4. Motivação } \\
\hline 4.10 texto é interessante; & 0,97 \\
\hline 4.2 Qualquer acompanhante que ler esse cartilha vai entender do que se trata ; & 0,95 \\
\hline 4.3 Você se sentiu motivado (a) a ler até o final; & 1,0 \\
\hline 4.4 O manual aborda os assuntos necessários ao familiar sobre a punção venosa; & 1,0 \\
\hline 4.5 O manual te ajuda a entender o passo a passo da punção venosa: & 0,97 \\
\hline $\begin{array}{l}\text { 4.6 A cartilha te ajuda a desenvolver estratégias para diminuir o estresse durante a } \\
\text { realização do procedimento; }\end{array}$ & 0,93 \\
\hline${ }^{* *}$ IAP Global & 0,97 \\
\hline
\end{tabular}

Fonte: Coleta de Dados

*IVC: Indice de Validação de Conteúdo

**IVC Global: Índice de Validação de Conteúdo Global

Em estudo realizado por Spagrud et al. (2008) apontou que os familiares que apresentavam atitudes positivas, as crianças tinham maiores índices de enfrentamento. Já os familiares que tiveram atitudes negativas, as crianças tinham maior estresse durante a CIP.

Segundo os familiares, que avaliaram a aplicabilidade prática da cartilha "Punção venosa periférica para família”, observou-se que essa auxilia a desenvolver estratégia que diminua o estresse, pois ela direciona como agir durante o procedimento, além de dar dicas do que fazer com a criança antes, durante e após a CIP.

Com relação ao conteúdo foi possível verificar que a cartilha aborda de maneira coerente os sentimentos vivenciados pelos familiares, esclarece o conceito de punção venosa, veia e cateter, e explica de maneira clara o passo a passo do procedimento e as estratégias podem ser adotadas pelos familiares durante a realização da punção venosa na criança. A linguagem e a ilustração são atrativas e facilitam o entendimento do assunto.

\section{CONCLUSÃO}

Diante disso, esse estudo possui uma elevada relevância científica, uma vez que, a cartilha torna-se um elemento importante para a produção do CCFC, sendo um instrumento facilitador no processo de fortalecimento dos vínculos entre a família e o profissional. Além de promover a segurança do paciente, pois a família torna-se e participante no processo de cuidado à criança.

\section{REFERÊNCIAS}

AVELAR, A. F. M.; PETERLINI, M. A. S.; PEDREIRA, M. L. G. 2013. Assertividade e tempo de permanência de cateteres intravenosos periféricos com inserção guiada por ultrassonografia em crianças e adolescentes. Rev. esc. enferm. USP 47( 3): 539-546. 
BENCHAYA, I; FERREIRA, E. A. P. BRASILIENSE, I. C. S. 2014. Efeitos de instrução e de treino parental em cuidadores de crianças com câncer. Psic.: Teor. e Pesq. [online] 30 (1): 13-23.

BEZERRA, A. R. et al. 2009 “ Minha punção venosa periférica”: um material didáticoinstrucional no preparo da criança para o procedimento. Rev. Soc. Bras. Enferm. Ped. 9 (2): 77-85. Disponível em: <http://www.sobep.org.br/revista/component/zine/article/120--minhapuno-venosa-perifrica-um-material-didticoinstrucional-no-preparo-da-criana-para-oprocedimento.html>. Acesso em: 20 jul. 2016.

CONCEIÇÃO, C. M. et al. 2011. Brinquedo terapêutico no preparo da criança para punção venosa ambulatorial: percepção dos pais e acompanhantes. Esc Anna Nery 15(2): 346-53. Disponível em: <http://www.scielo.br/pdf/ean/v15n2/v15n2a18.pdf>. Acesso em: 20 jul. 2016.

GRUPTA, H. V. et al. 2014. Comparison between the analgesic effect of two techniques on the level of pain perception during venipuncture in children up to 7 years of age: a quasiexperimental study. Journal of Clinical and Diagnostic Research 8(8):1-4.

LISBOA, S. D. 2014 Sentimentos de famílias de crianças hospitalizadas submetidas à punção intravenosa periférica (PIP): Subsídios para a construção de uma tecnologia didáticoinstrucional. 62 f. Monografia (Graduação em enfermagem) - Universidade Estadual de Feira de Santana, Feira de Santana.

LUPI, M.; PETTENGILL, A. M. 2008. Manual de orientações à família da criança sobre cuidados com cateter venoso central semi-implantado no domicilio. Rev. Soc. Bras. Enferm. Ped. São Paul. 8(2):73-81.

MAHONEY, L. AYERS, S; SEDDON P. 2010 The association between parent's and healthcare professional's behavior and children's coping and distress during venepuncture. $J$. Pediatr. Psychol 35(9):1-11.

PERROCA, M. G. 2011. Desarrollo y validación de contenido de la nueva versión de un instrumento para clasificación de pacientes. Rev. Latino-Am. Enfermagem 19(1): 58-66.

SCARPARO, A. F. et al. Reflexões sobre o uso da Técnica de Delphi em pesquisas na Enfermagem. Rev Rene. 13(1): 242-51.

SPAGRUD, L. J. et al. 2008. Pain, distress, and adult-child interaction during venipuncture in pediatric oncology: an examination of three types of venous access. Journal of Pain and Symptom Management. 36(2).

WHO, D. 2006. Effects of programmed information on coping behavior and emotions of mothers of young children undergoing iv procedures. Journal of Korean Academy of Nursing. 36(8). 